BULLETIN OF

MATHEMATICAL BIOLOGY

VOLUME 35,1973

\title{
ON ADJOINT DYNAMICAL SYSTEMS
}

\author{
Ion BăIAnu* \\ Faculty of Physics, \\ Biophysics Laboratory, \\ 14 Academiei Street, \\ Bucharest \\ Dragoș Scripcariu \\ Faculty of Biology, \\ Laboratory of Embryology and Cell Biology, \\ Splaiul Independenței nr. 92, \\ Bucharest
}

Transformations of dynamical systems are discussed in terms of adjoint, simple adjoint and weak adjoint functors. The relevance of this approach to interpretations of nuclear transplant experiments is suggested, and three new theorems concerning the development of biological systems are presented. Another three theorems concerning adjoint dynamical systems are proved. The connection of these results with the theory of organismic sets developed by Rashevsky (1966, 1967a-c, 1968a-c, 1969a-c, 1971a, b) is also investigated.

1. Introduction. In a previous paper (Băianu, 1971) a comparison of qualitative dynamics of systems was made. Also, new definitions of analogy and simple analogy between two dynamical systems were introduced, and they are formally different from those introduced by Rosen (1968). Consequently, it would be more convenient to use the terms "adjointness" and "simple adjointness" for denoting these concepts. The adjointness relations represent a certain similarity in the development of two systems.

One of the central problems of modern embryology is the manner in which W.8.

* Presently at Queen Elizabeth College, Physics Department, Campden Hill Road, London 
nucleocytoplasmic interactions determine cell differentiation during embryogenesis. For a recent review of these problems the reader is referred to Di Berardino and Hoffner (1970), and to Denis (1970). The main experimental approach to this problem was initiated by Briggs and King (1952) which developed a successful nuclear transplantation technique for the amphibian Rana pipiens. It must be remarked that these experiments are essentially relational, and that quantitative aspects of these experiments are of a secondary interest. Thus, it was possible by this technique to test for nuclear equivalence in cells. A series of studies of this type have shown that somatic nuclei from blastula stage to early stages of organogenesis become progressively restricted in their ability to promote normal development when transplanted into enucleated eggs. However, there are some other experiments which have shown that certain nuclei of blastula cells of Pleurodeles waltlii Michah are able to replace the zygote nucleus, subsequently producing a normal gastrulation and neurulation (Signoret and Picheral, 1962; Signoret, 1965). Also, some experiments of Smith (1965) show that a significant number of normal larvae of Rana pipiens was obtained from nuclei of primordial germ cells derived from stage 25 larvae. These last findings show that some nuclei of cells in certain different stages of development are equivalent. This equivalence can be abstractly represented by an isomorphism between the sets of temporal events in the two equivalent nuclei, together with an isomorphism between the sets of possible transformations (differentiations) of the equivalent totipotent nuclei. These mathematical requirements are rather strong and it may be that they are only an oversimplification of the real situations (see by the way the discussion and conclusion 4 in Burgess, 1967, pp. 38-39). However, on the basis of this abstract representation of nuclear equivalence, some interesting consequences can be mathematically derived, without having the pretence to explain all nuclear transplant experiments and all experimental results thus obtained. The above introduced isomorphisms are particular examples of an adjointness situation between equivalent nuclei of different cells. These mathematical requirements can be weakened in two distinct ways. The first is to consider isomorphisms restricted only to certain subsets of temporal events, and respectively, to certain subsets of possible transformations (differentiations). This corresponds to a simple adjointness relation (see Băianu, 1971) between the two dynamical systems. The second way is to substitute epimorphisms for isomorphisms in an adjointness relation. The concept which is thus obtained will be called weak adjointness. The next section will introduce precise mathematical definitions of adjointness, simple adjointness and weak adjointness. Then some consequences will be mathematically derived and their biological interpretations will be given. 
2. Adjointness and Limits. An adjointness relation between two dynamical systems implies the existence of a certain similarity in the development of the two systems. A dynamical system $D$ was previously defined by us as a commutative diagram which contains:

(i) the state space $X$ of $D$;

(ii) the aggregate of all temporal events in $D$ which produce the change from a given stage to the next;

(iii) the aggregate $\bar{S}$ of generating classes of $D$;

(iv) observables of $D$-defined as functors from $X$ to a category of $k$-tuples of real numbers, with $k=1,2, \ldots, n$.

(For further mathematical details see p. 556 in Băianu, 1970, hereafter referred to as I.)

This definition was introduced in order to account for development of a system, that is, for increases in the number of components, states and observables of the system in question. Thus, in our definition of a dynamical system, the "state space" $X$ is not fixed but varies according to certain rules of transformation. The number of possible states of $X$, as well as the structure of $X$, varies from one stage of development to the next. In earlier definitions of dynamical systems the state space, as well as its structure, were held fixed (Rosen, 1958). In our definition of a dynamical system the number of stages of development is determined by the transformation rules, and is fixed for a given dynamical system. Also, in a given initial stage the number of possible states is fixed. To each stage of development a generating class is associated. The generating classes of a dynamical system were previously defined in I (see pp. 546, 549) as triples of abstract categories together with the rules of transformation. They represent the functional connections among components in a given stage of development of a dynamical system (that is, the "organization" at that stage), in terms of diagrams of linked observables, together with the rules of transformation leading from that stage to the next. As an example, the $G_{1}$-stage of a cell can be abstractly represented by two generating classes-one corresponding to the nucleus, and the other corresponding to the cytoplasm of that cell. These generating classes are connected, although they can be separated (in the functional and in the physical sense). They were called "organismic classes". The initial organismic classes (which correspond to the $G_{1}$-stage), together with all organismic classes resulting from these by sequential transformations-form an aggregate which is called the generating organismic supercategory of the cell. Generating organismic supercategories were denoted in I by $S_{\sigma}$. 
The initial organismic class corresponding to a nucleus in the $G_{1}$-stage can be separated, as shown by nuclear transplant experiments. This separability property for organismic classes of zeroth order is expressed by the equality

$$
\left(C_{0}, \Pi, N_{0}\right)=\Pi_{0}
$$

with $C_{0}$ and $N_{0}$ being void, and with $\Pi_{0}$ corresponding to the organismic class assigned to the nucleus (see also axiom $K_{1}$ on p. 547 in I). Now, the precise definition of adjointness relation between two dynamical systems can be introduced.

$(D 1)$. Let $D$ and $D^{\prime}$ be two dynamical systems. Their aggregates of temporal events are denoted respectively by $T$ and $T^{\prime}$. Also denote by $\bar{S}$ and $\bar{S}^{\prime}$ the supercategories of generating classes of $D$, and respectively, of $D^{\prime} . \quad D$ is adjoint to $D^{\prime}$ if there exists:

(A) an isomorphism $T \stackrel{\sim}{\longrightarrow} T^{\prime}$;

$\left(A^{\prime}\right)$ two adjoint functors $\bar{S} \stackrel{K}{\longrightarrow} \vec{S}^{\prime} \stackrel{U}{\longrightarrow} \bar{S}$, with $K$ being the left adjoint of $U$.

The condition that $K$ is a left adjoint of $U$ is equivalent to the existence of the isomorphisms

$$
\bar{S}[A, U(B)] \stackrel{\sim}{\longrightarrow} \bar{S}^{\prime}[K(A), B]
$$

with $A$ being an arbitrary object in $\bar{S}$ and with $B$ being an arbitrary object in $\bar{S}^{\prime}$ (Kan, 1958). $\quad U(B)$ is the image of $B$ in $\bar{S}$ under the action of $U$, and $K(A)$ is the image of $A$ in $\bar{S}^{\prime}$ under the action of the functor $K . \quad \bar{S}[A, U(B)]$ represents the set of all possible transformations in $\bar{S}$ from the generating class $A$ to the generating class $U(B) . \quad \bar{S}^{\prime}[K(A), B]$ represents the set of all possible transformations in $\bar{S}^{\prime}$ from the generating class $K(A)$ to the generating class $B$. The isomorphism in (2) represents the equivalence between $A$ and $K(A)$, that is, it shows that the number of possible transformations of $A$ in $\bar{S}$ is equal to the number of the possible transformations of $K(A)$ in $S^{\prime}$. It may be also said that $A$ is totipotent if $K(A)$ is so.

(D2). Let $\bar{S}^{\circ}$ be a full subsupercategory of $\bar{S}^{\prime}$, from $D^{\prime}$. Two dynamical systems $D$ and $D^{\prime}$ are simply adjoint if there exists an isomorphism $T \stackrel{\sim}{\longrightarrow} T^{\circ}$, with $T^{\circ} \hookrightarrow T^{\prime}$, and two adjoint functors $\bar{S} \stackrel{M}{\longrightarrow} \bar{S}^{\circ} \stackrel{L}{\longrightarrow} \bar{S}$, with $M$ being a left adjoint of $L$.

This definition introduces in fact an adjointness relation between a dynamical system $D(T, \bar{S})$ and a dynamical system $D^{\circ}\left(T^{\circ}, \bar{S}^{\circ}\right)$, which is induced by $\bar{S}^{\circ}$.

(D3). Two dynamical systems $D$ and $D^{\prime}$ are called weakly adjoint if there exist an epimorphism $T \longleftrightarrow T^{\prime}$ and two weak adjoint functors $\bar{S} \stackrel{W}{\longrightarrow} \bar{S}^{\prime} \stackrel{V}{\longrightarrow} \bar{S}$. 
The condition that $W$ and $V$ are weak adjoint functors means that there exists an epimorphism

$$
\bar{S}[X, V(Y)] \succ \bar{S}^{\prime}[W(X), Y]
$$

for any $X$ in $\bar{S}$, and any object $Y$ in $\bar{S}^{\prime}$, (Kainen, 1971). A weak adjoint is not necessarily a proper functor, that is, it may be multi-valued on morphisms.

Adjoint functors of supercategories can be introduced in a different way by natural equivalences (functorial isomorphisms), as follows. If $C$ is some category in $\bar{S}$, and if $C^{\prime}$ is a category in $\bar{S}^{\prime}$, then consider the functor $\operatorname{Hom}_{C}(U)$ : $C^{\circ} \times C^{\prime} \longrightarrow$ Ens, with $C^{\circ}$ being the dual of $C$ and with Ens being the category of all sets and maps among sets. The functor $\operatorname{Hom}_{C}(U)$ associates with each object $(A, B)$ in $C^{\circ} \times C^{\prime}$ a set $C[A, U(B)]$ of morphisms of $C$, with $U: C^{\prime} \longrightarrow C$ being a certain functor which carries $B$ from $C^{\prime}$ to $U(B)$ in $C$. Similarly, define a functor $\operatorname{Hom}_{C^{\prime}}(K): C^{\circ} \times C^{\prime} \longrightarrow$ Ens which associates to each object $(A, B)$ of $C^{\circ} \times C^{\prime}$ the set $C^{\prime}[K(A), B]$, with $K: C \longrightarrow C^{\prime}$ being a functor. In these conditions, $K$ is a left adjoint of $U$ if there exists a natural equivalence

$$
\varphi: \operatorname{Hom}_{C^{\prime}}(K) \longrightarrow \operatorname{Hom}_{C}(U)
$$

(Bucur and Deleanu, 1968).

Now, if $C$ is varied in $\bar{S}$, and if $C^{\prime}$ is varied in $\bar{S}^{\prime}$, then adjoint functors of supercategories

$$
\bar{S} \stackrel{K}{\longrightarrow} \bar{S}^{\prime} \stackrel{\sigma}{\longrightarrow} \bar{S}
$$

are induced by the underlying natural equivalences (4). Equivalently, it will be denoted as

$$
\varphi: \operatorname{Hom}_{S}(K) \longrightarrow \operatorname{Hom}_{S^{\prime}}(U),
$$

where $\varphi$ is explicitly determined by the family

$$
\left\{\varphi_{i}: \operatorname{Hom}_{C_{i}}\left(K_{i}\right) \longrightarrow \operatorname{Hom}_{C_{i}^{\prime}}\left(U_{i}\right)\right\},
$$

with $C_{i}$ varying in $\bar{S}$, and $C_{i}^{\prime}$ varying in $\bar{S}^{\prime}\left(K_{i}, U_{i}\right.$ and $\varphi_{i}$ vary following $C_{i}$ and $C_{i}^{\prime}$ ). From the above definition the following statement is directly obtained.

\section{Proposition 1}

If an adjointness relation exists between two dynamical systems, then it is unique up to an isomorphism.

Since the unicity of adjointness relation is shown we can now proceed to present some results concerning adjoint dynamical systems.

\section{Theorem 1}

Let $D$ and $D^{\prime}$ be two adjoint dynamical systems and consider the two functors 
$\bar{S} \stackrel{K}{\longrightarrow} \bar{S}^{\prime} \stackrel{J}{\longrightarrow} \bar{S}$ which are present in $\left(A^{\prime}\right)$ of $(D 1)$. Then, the following assertions are valid:

(1) $K$ preserves inductive superlimits of $\bar{S}$;

(2) $U$ preserves projective superlimits of $\bar{S}^{\prime}$.

\section{Proof}

The result is obtained on the basis of condition (5). The underlying functors $K$ preserve inductive limits (colimits) of the categories $C_{i}$. Also, the underlying functors $U$ preserve projective limits (or limits) of $C_{i}^{\prime}$ (see Theorem 5.5, on p. 19 in Popescu, N., 1971). The existence of supercolimits (injective superlimits) or of superlimits in $\bar{S}^{\prime}$ is ensured by the existence of colimits in categories $C_{i}$ of $\bar{S}$, and respectively, by the existence of limits in categories $C_{i}^{\prime}$ of $\bar{S}^{\prime}$. One also has that $K$ preserves all colimits in $\bar{S}$ through the simultaneous action of the underlying functors $K$. Similarly, $U$ preserves all limits in $\bar{S}^{\prime}$ through the actions of underlying functors $U$. Consequently, $K$ preserves supercolimits of $\bar{S}$, and $U$ preserves superlimits of $\bar{S}^{\prime}$. Q.E.D.

The following corollary introduces an even stronger condition on adjoint functors, and it is a consequence of certain corresponding algebraic properties of adjoint functors of categories.

\section{Corollary 1}

Let $F: \bar{S} \longrightarrow \bar{S}^{\prime}$ be a functor of supercategories, and let $G$ be its right adjoint. Also, consider $G: \bar{S}^{\prime} \longrightarrow \bar{S}$ to be full and faithful. Then the two following assertions are true:

(i) if $\bar{S}$ has projective superlimits then $\bar{S}^{\prime}$ also has superlimits;

(ii) if $\bar{S}^{\prime}$ has inductive superlimits then $\bar{S}$ also has supercolimits.

\section{Proof}

The result is obtained on the basis of Corollary 5.6 of Popescu, N. (1971, p. 19), and the reasoning is similar to that of the previous theorem.

\section{Note}

The above results are referring only to generating supercategories $\bar{S}$ of adjoint dynamical systems, while Proposition 3 in I is referring to the "state space" $X_{\sigma}$ (organismic supercategory) of biological systems which is subject to the principle of choice (asserting the existence of limits or colimits in organismic supercategories). The relation between $X_{\sigma}$ and $S_{\sigma}$ for adjoint biological systems is shown by the following theorem.

\section{Theorem 2}

Let $D_{\sigma}$ and $D_{\sigma}^{\prime}$ be two adjoint biological systems. The superpushout of the 
organismic supercategory $X_{\sigma}^{\prime}$ (associated with $D_{\sigma}^{\prime}$ ), is carried on a corresponding superpushout of the organismic supercategory $X_{\sigma}$-which is associated with $D_{\sigma}$, if and only if the right adjoint $G: X_{\sigma}^{\prime} \longrightarrow X_{\sigma}$ of $F: X_{\sigma} \longrightarrow X_{\sigma}^{\prime}$ is full and faithful.

\section{Proof}

Corollary 1 holds for any supercategory and, in particular, it is true for organismic supercategories. We have only to use statement (ii) of this corollary since the presence of inductive limits (superlimits) ensures the existence of pushouts (superpushouts). As in Proposition 2 of I, Corollary 1 requires that $G: X_{\sigma}^{\prime} \longrightarrow X_{\sigma}$ be full. In addition, it requires that $G$ be faithful. Proposition 3 in $I$ (see p. 559) requires the existence of a coadjoint $L:[\bar{\Sigma}, X] \longrightarrow X$ of $I: X \longrightarrow[\bar{\Sigma}, X]$, with $[\bar{\Sigma}, X]$ being the class of all superdiagrams in $X$ over the superscheme $\Sigma$. The construction of the functor $I$ is realized by considering $\bar{S}_{\sigma}$ to be a superscheme of diagrams in $X_{\sigma}$. Then we get I: $X \longrightarrow\left[\bar{S}_{\sigma}, X_{\sigma}\right]$, and $L:\left[\bar{S}_{\sigma}, X_{\sigma}\right] \longrightarrow X_{\sigma}$ (the coadjoint of I). Similarly, one has certain functors $I^{\prime}:\left[\bar{S}_{\sigma}^{\prime}, X_{\sigma}^{\prime}\right] \longrightarrow X_{\sigma}^{\prime}$. The theorem is proved by the fact that the following diagram is commutative

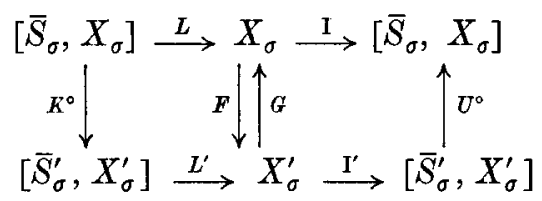

where one actually needs only the right square of this diagram. In the above diagram $K^{\circ}$ is induced by $K: \bar{S}_{\sigma} \longrightarrow \bar{S}_{\sigma}^{\prime}$, and by $F: X_{\sigma} \longrightarrow X_{\sigma}^{\prime}$. Similarly, $U^{\circ}$ is induced by $U: \bar{S}_{\sigma}^{\prime} \longrightarrow \bar{S}_{\sigma}$ and by $G: X_{\sigma}^{\prime} \longrightarrow X_{\sigma}$. $G$ is the functor which conforms to conditions of Corollary 1 , and which, as a consequence of the above construction, carries a superpushout of $X_{\sigma}^{\prime}$ on a corresponding superpushout of $X_{\sigma}$. Q.E.D.

Theorem 1 and Corollary $l$ apply in a specific manner to the theory of organismic sets and to the theory of $(M, R)$-systems. For applications concerning $(M, R)$-systems see Băianu (1973) and Băianu and Marinescu (1973).

It was shown in I that any category $S_{0}^{S}$ of functors, from the discrete category $S_{\mathrm{o}}$ (organismic set) to a certain algebraic theory $S$, has limits and colimits (see the theorem on p. 522). These functors define a kind of coarse observables of organismic sets. They assign to each element $e_{i}$ of an organismic set $S_{\mathrm{o}}$ (discrete category), the corresponding sets of its activities, products, or intensities of these activities and quantities of products. Adjoint functors between categories of the type $S_{0}^{S}$ preserve limits or colimits. A particular example of colimit is given here in order to get an intuitive picture of such an abstract situation. 
Consider that the first stage of development of an organism is represented by an organismic set $S_{0}^{1}$. The next stage would be represented by a larger organismic set $S_{0}^{2}$, and so on. These organismic sets form a directed system if we assume that there exist the canonical inclusions

$$
S_{\mathrm{o}}^{1} \stackrel{r_{12}}{\longrightarrow} S_{0}^{2} \hookrightarrow \cdots \hookrightarrow S_{0}^{n} \stackrel{r_{n 0}}{\longrightarrow} S_{0} .
$$

A directed system $A$ is a commutative diagram over a scheme $I$, which is a directed set. A morphism in $A$ is drawn between objects $A_{i}$ and $A_{j}$ if $i \leqq j$, with $i, j \in I$. Commutativity of the diagram requires that if $i \leqq j$ there is only one morphism $A_{i} \stackrel{r_{i j}}{\longrightarrow} A_{j}$ in $A$, such that $r_{j k} \circ r_{i j}=r_{i k}$ for $i \leqq j \leqq k$, and $r_{i i}=1_{A_{i}}$, for all $i \in I$ (Mitchell, 1965). The direct limit $L$ of this directed system is the set which is the disjoint union of all preceding sets, and is denoted by $\underset{i \in I}{\longrightarrow}{ }^{A_{i}}$. An intuitive image of the above introduced directed system of organismic sets is given in Figure 1, where the direct limit $S_{0}$ is indicated by the outer circle. Naturally, this situation is only an oversimplification of the development of an organismic set, and is only a particular case of a colimit. In the same manner, a directed system $R$ of sets of relations can be defined. Consider these relations to be those which appear as an organismic set develops. It is obvious that the principle which determines the course of development of an organismic set (Rashevsky, 1969c) can be restated by requiring that the directed limit of $R$ does exist. Then, the direct limit of $R$ is the maximal set of relations of the developing organismic set (it is in fact the disjoint union of sets of relations in $R$ ).

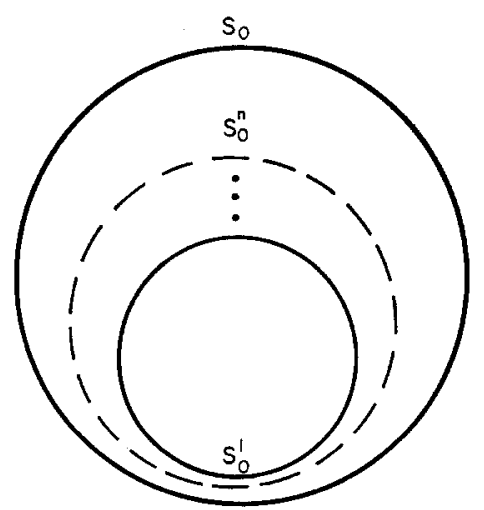

Figure 1. The direct limit $S_{\mathrm{o}}$ of organismic sets $S_{0}^{1}, \ldots, S_{0}^{n}$

Since a direct limit is a particular realization of a colimit, the existence of direct limits in some organismic supercategories is assured by the principle of choice (Băianu and Marinescu, 1968). Remark that the variety of relations in 
$R$ is maximized in the direct limit. It can be easily proved that a particular realization of our theorem in I-showing the existence of projective limits in $S_{0}^{S}$-implies that one can define the set of observables of an organismic set $S_{0}$ as the cartesian product of the sets of observables of its elements $e_{i}$. This is justified by the fact that, in a category of sets, the projective limit of a family of sets can be introduced as the cartesian product of the sets in that family. If one transforms an organismic set $S_{0}^{\prime}$ into an organismic set $S_{0}$, then the set of observables of $S_{\circ}^{\prime}$ is isomorphically mapped on the set of corresponding observables of $S_{0}$, if and only if there exist adjoint functors $K: S_{0}^{S} \longrightarrow S_{0}^{\prime S^{\prime}}, S_{\mathrm{o}}^{\prime S^{\prime}} \longrightarrow$ According to Theorem 1, $U$ preserves projective limits of $S_{0}^{\prime \prime^{\prime}}$, that is, $U$ maps the set of all observables of $S_{\mathrm{o}}^{\prime}$ on the set of corresponding observables of $S_{0}$. In the case of a simple adjointness between $S_{0}^{\prime S^{\prime}}$ and $S_{0}^{S}$, the set of observables of the organismic set $S_{\mathrm{o}}^{\prime}$ is isomorphic to a subset of observables of the organismic set $S_{0}$.

3. Adjointness Theorems for Nuclear Equivalence. The following result refers to weakly adjoint dynamical systems, and is a direct consequence of the realization theorem of Kainen (1971), (see Theorem and Corollary on p. 8).

\section{Theorem 3}

Let $D$ and $D^{\prime}$ be two weakly adjoint dynamical systems. Suppose that the generating supercategory $S$ of $D$ has weak pushouts. Also suppose that the weak, right adjoint $U: \bar{S}^{\prime} \longrightarrow \bar{S}$ of $K: \bar{S} \longrightarrow \bar{S}^{\prime}$ preserves weak pushouts. In these conditions, given any commutative square diagram $q$ in $\bar{S}$, there exists a commutative square diagram $q^{\prime}$ in ${\overline{S^{\prime}}}^{\prime}$ such that $U\left(q^{\prime}\right)=q$ if $U \circ K=1_{\bar{s}}$.

The commutative diagrams can be considered to represent equivalent possible ways in which the nuclei may develop. In the case of nuclear transplants, weak adjoint "functors" map the possible equivalent developments of the transplanted nucleus onto the possible equivalent developments of the replaced nucleus. This equiralence may be preserved until a limiting given stage of development of the embryo. After that stage the transplanted nuclei would produce sharp differences in development as compared to the normal course of development. Experiments indicate blastula stage to be the limiting stage.

\section{Theorem 4}

Let $D$ be a dynamical system and let $\bar{S}$ be its generating supercategory. Suppose that $\bar{S}$ has projective superlimits, that is, all categories $C_{t}$, in $\bar{S}$ have projective limits. If $C_{k}$ is an arbitrary category of $\bar{S}$ then consider that for any $A$ in $C_{k}$ the subobjects of $A$ form a set. If $C_{k}$ are small full subcategories of the corresponding $C_{k}$ then there exist full subcategories of $C_{k}^{\hat{\alpha}}$ of $C_{k}$ so that: 
(i) the inclusion functors $C_{k}^{\circ} \hookrightarrow C_{k}^{\hat{k}}$ preserve inductive limits and cogenerate $C_{k}^{\hat{k}}$;

(ii) all $C_{k}^{\wedge}$ have inductive and projective limits, and all inclusion functors $C_{\hat{k}}^{\wedge} \hookrightarrow C_{k}$ have left adjoints;

(iii) the generating supercategories $S^{\wedge}$, induced by the categories $C_{k}^{\wedge}$, lead to the generation of a dynamical system $D^{\wedge}$, such that $D$ and $D^{\wedge}$ are adjoint.

\section{Proof}

The first two sentences are only reformulations of a result of Popescu, D. (1970). The third results from the second sentence by taking into account the definition of adjointness and the definition of adjoint "functors" of supercategories.

A particular case of the above theorem is realized by the nuclear clones (King and Briggs, 1956) when the generating classes, which represent nuclei in a serial transplantation experiment, lead to generating supercategories which are adjoint. (In fact, it is the dynamical systems, induced by these generating supercategories, which are the adjoint entities.) It may be easily checked that theother conditions of the theorem are fulfilled for nuclear clones. The fact that all nuclei in the clone result in the same type of development appears as a consequence of particular adjointness relations between nuclei which are subject to the conditions of the above theorem.

Without any further restrictions it would be difficult to obtain results concerning weak adjoint nuclei. The addition of the principle of relational invariance (Rashevsky, 1968b), as a restriction to the weak adjointness conditions for the transplanted and replaced nuclei, points toward the following theorem.

\section{Proposition 2}

The nucleus of a fertilized ovum is weak adjoint with any other nucleus, directly, or indirectly, resulting from it.

This proposition states in fact that all possible transformations of the generating classes of the fertilized ovum are mapped onto the possible transformations of any nucleus resulting from the nucleus of the fertilized ovum.

\section{Corollary 2}

Any nucleus has the potential ability of inducing the self-reproduction (division) of cells.

This corollary is a particular realization of Proposition 2 since one of the most 
important possible transformations of a nucleus is the one leading to DNA reduplication, and to subsequent replication of chromosomes. The corollary states that transformations of a nucleus which lead to DNA synthesis aremapped onto corresponding transformations of any nucleus which results as a consequence of sequential divisions of the fertilized ovum. This statement is in agreement with the axiomatic introduction of self-reproducing entities in (D1) of I (see p. 546), and with the fact that only some pairs of such entities can be eventually transformed into non-reproducible entities. Experimental results of Graham et al. (1966) and of Gurdon (1967) show that this is the case. They have experimentally proved - by means of nuclear transplant experiments - that nuclei of brain cells (which never divide inside the brain) begin to synthetize DNA immediately after their implantation in the oocyte wall.

The results of this section are referring only to transformations which occur at the functional-physiological level. It becomes of greater and greater interest to have a fruitful approach to the molecular and submolecular levels of organization of the transplanted nuclei. Therefore it would be interesting to study theoretically the adjointness relations which exist at the molecular and quantum levels. This is the aim of a subsequent paper.

The authors would like to devote this paper to the memory of Professor Nicolas Rashevsky who, in more than one way, initiated this approach.

\section{LITERATURE}

Băianu, I. 1970. "Organismic Supercategories: II. On Multistable Systems." Bull. Math. Biophysics, 32, 540-561.

. 1971. "Organismic Supercategories and Qualitative Dynamics of Systems." Ibid., 33, 339-354.

General Unitary Theory of Systems." Ibid., 30, 625-636.

- and 1973. "A Functorial Construction of $(M, R)$-Systems." Rev. Roum. Math. Pures et Appl. (in press).

1973. "Some Algebraic Properties of $(M, R)$-Systems." Bull. Math. Biology, 35, 213-217.

Briggs, R. and T. King. 1952. "Transplantation of Living Nuclei from Blastula Cells into Enucleated Frogs' Eggs." Proc. Natl. Acad. Sci. U.S., 38, 455-463.

Bucur, I. and Deleanu, A. 1968. Introduction to the Theory of Categories and Functors, pp. 16-52. London, New York, Sydney: Wiley.

Burgess, M. A. 1967. "The Developmental Potentialities of Regeneration Blastema Cell Nuclei as Determined by Nuclear Transplantation." J. Embryol. Exp. Morph., 18, $27-41$.

Denis, H. 1970. "Importance des Interactions Nucleo-Cytoplasmiques au Cours du Développement Embryonnaire." Arch. Int. Physiol. Biochim., 78, 367-380.

Dỉ Berardino, A. M. and Nancy Hoffner. 1970. "Origin of Chromosomal Abnormalities in Nuclear Transplants-A Reevaluation of Nuclear Differentiation and Nuclear Equivalence in Amphibians." Develop. Biol., 23, No. 2, 185-209. 
Graham, C. F. et al. 1966. "The Induction of DNA Synthesis by Frog Egg Cytoplasm." Develop. Biol., 14, 349-381.

Gurdon, J. B. 1967. "On the Origin and Persistence of a Cytoplasmic State Inducing Nuclear DNA Synthesis in Frogs' Eggs." Proc. Natl. Acad. Sci. U.S., 58, 545-552.

Kainen, P. 1971. "Weak Adjoint Functors." Math. Z., 122, 1-9.

Kan, D. M. 1958. "Adjoint Functors." Trans. A.M.S., 87, 294-330.

King, T. J. and R. Briggs. 1956. "Serial Transplantation of Embryonic Nuclei." Cold Spring Harbor Symp. Quant. Biol., 21, 271-290.

Mitchell, Barry. 1965. The Theory of Categories. New York and London: Academic Press.

Popescu, D. 1970. "Some Remarks on Complete Cogenerated Categories." Rev. Roum. Math. Pures et Appl., 15, 1027-1033.

Popescu, N. 1971. Abelian Categories (in Romanian). Bucharest: Ed. Academy of R.S. Romania.

Rashevsky, Nicolas. 1966. "Physics, Biology and Sociology: A Reappraisal." Bull. Math. Biophysics, 28, 283-308.

- 1967a. "Organismic Sets: Outline of a General Theory of Biological and Social Organisms." Ibid., 29, 139-152.

- 1967b. "Physics, Biology and Sociology: II. Suggestions for a Synthesis." Ibid., 29, 643-648.

-. 1967c. "Organismic Sets and Biological Epimorphism." Ibid., 29, 389-393.

. 1968a. "Organismic Sets: II. Some General Considerations." Ibid., 30, $163-173$.

- 1968b. "Neurocybernetics as a Particular Case of General Regulatory Mechanisms in Biological and Social Organisms." Concepts de l'Age de la Science, 3, 243-258.

- 1968c. "A Note on the Development of Organismic Sets." Bull. Math. Biophysics, 30, 355-358.

- 1969a. "Outline of a Unified Approach to Physics, Biology and Sociology." Ibid., 31, 159-198.

- 1969b. "Multiple Relational Equilibria: Polymorphism, Metamorphosis and Other Possibly Similar Phenomena." Ibid., 31, 417-427.

-1969c. "Some Considerations of Relational Equilibria." Ibid., 31, 605-617.

-1971a. "Two Definitions of Stability of Equilibria." Ibid., 33, 157-164. 321-338.

Rosen, Robert. 1958. "The Representation of Biological Systems from the Standpoint of the Theory of Categories." Ibid., 20, 317-342.

-1968. "On Analogous Systems." Ibid., 30, 481-492.

2. 1971. "Some Realizations of $(M, R)$-Systems and Their Possible Interpretations." Ibid., 33, 303-320.

Signoret, J. and B. Picheral. 1962. "Transplantations de Noyaux chez Pleurodeles Waltlii Michah." C.R. Acad. Sci. Paris, 254, 1150-1151.

-1 1965. "Transplantations Nucléaires et Différenciation Embryonnaire." $A r$ chives de biologie (Liege), 76, 591-606.

Smith, L. D. 1965. "Transplantation of Nuclei of Primordial Germ Cells into Enucleated Eggs of Rana pipiens." Proc. Nat. Acad.Sci. U.S., 54, 101-107.

RECEIVED 1-8.72 